\title{
Wave Activity and Prominence Eruption
}

\author{
F. Baudin, K. Bocchialini
}

Institut d'Astrophysique Spatiale/Univ. Paris XI Bât. 121, Campus d'Orsay, 91405 Orsay, France

C. Delannee, S. Koutchmy, G. Stellmacher

Institut d'Astrophysique de Paris/CNRS, 98 Bis Bd Arago, 75014 Paris, France

K. Shibata

National Astronomical Observatory, Mitaka 181 Tokyo, Japan

I.S. Veselovsky, O.A. Panasenko, A.N. Zhukov

Institute of Nuclear Physics, Moscow State University, Moscow 11989g, Russia

Abstract. Observational evidence of 3 and 5 min vertical oscillations of a filament on the disk are recorded. Wave activity was observed before, during and after a filament disappearance, inside and around the filament. Both an $\mathrm{H}_{\alpha}$ brightening and, later, a blowing out of a faint soft X-ray (Yohkoh) loop system occuring in connection with a flare were noticed. The wave activity seems to be a dynamically important ingredient of this erupting prominence.

Propagating MHD waves and convective structures bring their energy and momentum from the photosphere towards the chromosphere up to the coronal heights where they are partially reflected and/or dissipated. The transition from the laminar to the turbulent state of the whole prominence enhances the dissipation rate of the external waves inside this system, adding energy to produce the heating and lifting of the plasma. Internal plasma instabilities could trigger this transition in the framework of a prominence disappearance.

\section{Introduction}

Conditions for prominence formation are thought to be related to the arcade of coronal fields joining opposite-polarity photospheric fields with the convergence and cancellation of small patches of opposite-polarity magnetic flux (Martin 1990). The concept of "the ball in the air" supported in a statistical sense by nonlinear Alfvén waves has been developed for quiescent prominences (Jensen 1990). Nonlinear oscillations of the plasma near the tops of magnetic loops in the gravity field could make this configuration dynamically stable (Gorbachev and Kelner 1988). A connection between the filament and the chromospheric 
network was suggested based on the observed up- or downward motions up to $\sim 10 \mathrm{~km} / \mathrm{s}$ at the footpoints (Schmieder 1990). MHD shock waves travelling along fibrils were suggested as a possible explanation of this connection (Sterling and Hollweg 1988).

Prominence oscillations have been reported in many papers (see, e.g., Bashkirtsev and Mashnich 1984, Wiehr et al. 1989, Molowny et al. 1997). The viscous friction or the wave emission are supposed to be important dissipative factors in the late phase of an erupting prominence. The aim of the present paper is to present new observations of oscillations in a disappearing filament on the disk and to discuss a possible role of small-scale processes in the phenomenon.

\section{Observations}

The observations were obtained at the Sacramento Peak Observatory Vacuum Tower Telescope (VTT). Sequences of images in the $\mathrm{H}_{\alpha}$ red and blue wings (at $0.75 \AA$ from the line center) taken through the narrow UBF passband were obtained during $110 \mathrm{~min}$ with a sampling time of $1 \mathrm{~min}$, an exposure time of $0.8 \mathrm{~s}$, and a spatial resolution of $0.34 \mathrm{arcsec} / \mathrm{px}$ [see details in Baudin et al. (1997), who also made observations in the continuum and the $\mathrm{MgI}_{b 1}$ line in the same field of view]. Here, we concentrate on the analysis concerning a filament lying between plages with opposite polarities.

We observed a very rapid temporal evolution of the filament, which moved over an apparent distance of $18,000 \mathrm{~km}$ in $12 \mathrm{~min}$ (between $\mathrm{T}_{\text {obs }}=42$ and $54 \mathrm{~min}$, leading to a minimum speed of $25 \mathrm{~km} / \mathrm{s}$, see Figure 1), which was followed by the eruption of a loop system observed in soft X-rays by the Yohkoh telescope about 2 hours later. We observed also oscillations in the $2-8 \mathrm{mHz}$ range in the region of the filament over the whole time sequence, but stronger "pulses" of power (aroud 3 and $5.5 \mathrm{mHz}$ ) occurred at the beginning of the eruption of a large part of the filament (see Figure 1) in the time/frequency analysis of a time series extracted from a region at the center of the filament.

\section{Discussion}

Prominences show numerous manifestations of the mechanical and thermodynamical loss of equilibrium. Faint dissipative MHD and kinetic properties of the gas and electromagnetic radiation seem to be important in the regulation of the energy, momentum and mass flows associated with filaments and prominences. It is generally believed that MHD waves and explosive events are essential phenomena (Jensen 1990). Many important details are not known, so we can only guess about dominant MHD and kinetic processes.

For these purposes we used estimated parameters characterizing filaments. We distinguished three more or less arbitrary scales in filaments: the largest characteristic scales for the structure as a whole (L), substructures (M) and the smallest (S) observable scales (see Table 1). Several implications follow from the analysis of physical conditions. Both thermal and bulk motions can be essential in the prominence structure and dynamics. The corresponding MHD regimes can be nearly sonic because $M \sim 1$. The cool parts $\left(T<10^{4}\right)$ are 

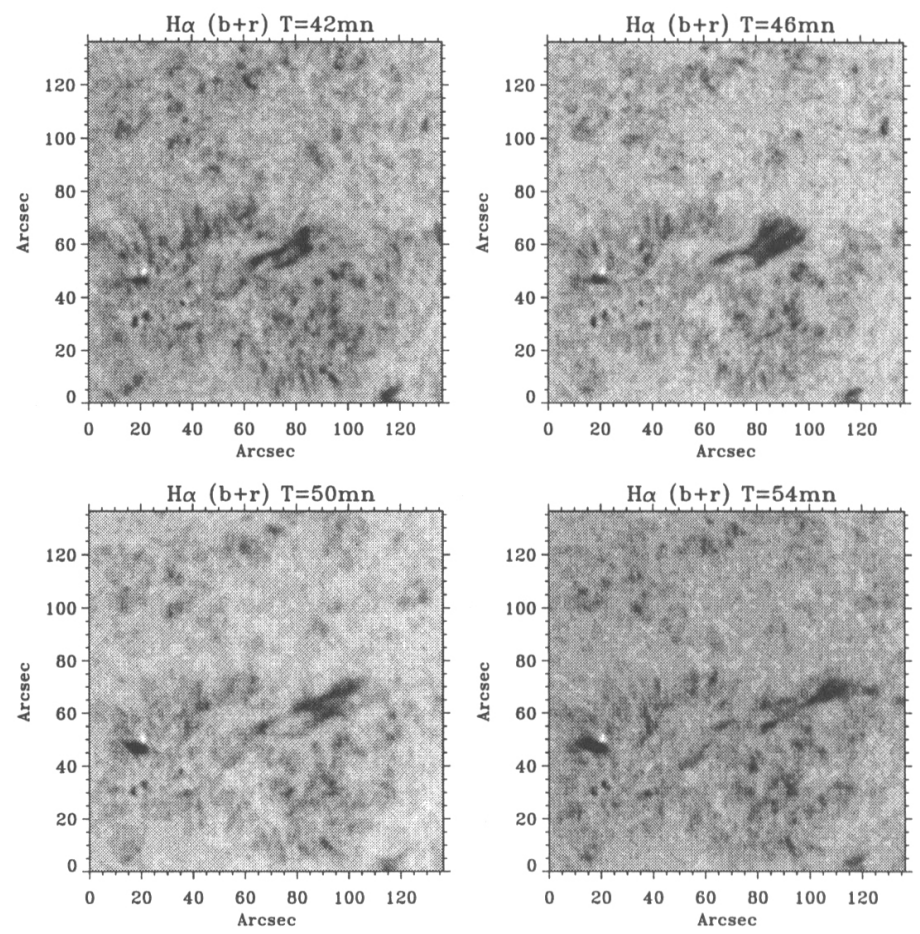

Figure 1. Eruption of the filament as seen in frames with both $\mathrm{H}_{\alpha}$ wings added together, during a period of time of $12 \mathrm{~min}$.

probably dominated by radiation in the energy balance. Radiative $\mathrm{H}_{\alpha}$ losses are comparable to the estimated MHD wave energy inputs. Viscous terms may be neglected because $R e \gg 1$. This means that fows are turbulent for these scales. The analysis of dimensionless parameters shows that $\mathrm{L}$ structures are quasistationary. This is not the case for $M$ and $S$ structures. High magnetic Reynolds numbers allow us to use the frozen-in approximation. Motions along magnetic fields in prominences and filaments are regulated by the combined action of the gas pressure and gravity forces with the inertia term due to the inhomogeneity of the drifts across the magnetic field. As a result, magnetoacoustic-gravity waves and convective structures appear.

Alfvén waves are nonlinearly damped in the low magnetic field, dense regions. Previous work (Suess et al. 1992) stated that 5 min Alfvén waves are not reflected in the solar corona up to heights of about 6 solar radii, but this could be different in the presence of sharp inhomogeneities. Small-scale structures inside filaments and prominences can reflect and scatter the waves coming from the bottom. An increase of the turbulence level due to the internal instability adds to the scattering and the dissipation of the external waves because of the appearance of new scattering and absorbing elements. We speculate that the transition to a more turbulent state of the prominence produces an enhancement of the overall dissipation of the external energy fluxes crossing the system 
in the shape of quasi-stationary electric currents and mechanical motions, as well as waves ( 3 and 5 min oscillations). The enhanced dissipation probably leads to the brightening, heating, and proper oscillations of the prominence, and eruption when the lifting force is large enough.

Table 1. The characteristic scales in filaments and prominences

\begin{tabular}{|c|c|ccc|c|}
\hline & & \multicolumn{3}{|c|}{ Dimension (Mm) } & Lifetime \\
\cline { 3 - 5 } & & Length & Height & Width & \\
\hline L & Large scale ranges & $60-600$ & $15-100$ & $4-15$ & weeks-months \\
\hline M & Medium scale & \multicolumn{3}{|c}{30} & $2-12$ hours \\
\hline S & Small scale & & $0.3-1$ & $\leq 10$ min \\
\hline
\end{tabular}

\section{Conclusion}

Our results lend some additional support to the view that the role of waves and small-scale convective structures in filaments and prominences may be twofold. First, they bring energy and momentum from the photosphere towards the chromosphere up to coronal heights where they are partially reflected and dissipated. Second, the fast transition from a quiescent to the eruptive state could be triggered by the switch of the extreme gas pressure gradient or the electric currents or by a rapid increase of internal small-scale plasma inhomogeneities, modifying the dissipative characteristics of the prominence/cavity plasma system.

\section{References}

Bashkirtsev V.S. and Mashnich G.P. 1984, Solar Phys., 91, 93

Baudin F., Molowny-Horas R. and Koutchmy, S. 1997, A\&A, 326, 842

Gorbachev V.S. and Kelner S.R. 1988, Zh. Exp. Theor. Phys., 94 (9), 89

Jensen E. 1990, in Dynamics of Quiescent Prominences, V. Ruždjak and E. Tandberg-Hanssen (eds.), Springer-Verlag, New York, p. 129

Martin S.F. 1990, in Dynamics of Quiescent Prominences, V. Ruždjak and E. Tandberg-Hanssen (eds.), Springer-Verlag, New York, p. 1

Molowny-Horas R. et al. 1997, Solar Phys., 172, 181

Schmieder B. 1990, in Dynamics of Quiescent Prominences, V. Ruždjak and E. Tandberg-Hanssen (eds.), Springer-Verlag, New York, p. 85

Sterling A.C. and Hollweg J.V. 1988, ApJ, 327, 950

Suess S.T., Moore R.L., Musielak Z.E. and An C-H. 1992, in Solar Wind Seven, E. Marsch and R. Schwenn (eds.), Pergamon Press, Oxford, p. 117

Wiehr E., Balthasar H. and Stellmacher, G. 1989, Hvar Obs. Bull., 13 (1), 131 